\title{
Multiscale quantum-defect theory for two interacting atoms in a symmetric harmonic trap
}

\author{
Yujun Chen and Bo Gad* \\ Department of Physics and Astronomy, University of Toledo, MS 111, Toledo, Ohio 43606
}

(Dated: April 19, 2018)

\begin{abstract}
We present a multiscale quantum-defect theory (QDT) for two identical atoms in a symmetric harmonic trap that combines the quantum-defect theory for the van der Waals interaction [B. Gao, Phys. Rev. A 64, 010701(R) (2001)] at short distances with a quantum-defect theory for the harmonic trapping potential at large distances. The theory provides a systematic understanding of two atoms in a trap, from deeply bound molecular states and states of different partial waves, to highly excited trap states. It shows, e.g., that a strong $p$ wave pairing can lead to a lower energy state around the threshold than a $s$ wave pairing.
\end{abstract}

PACS numbers: 03.75.Nt,34.10.+x,03.65.Ge,32.80.Pj

\section{INTRODUCTION}

Two interacting particles under confinement, described generally by a Hamiltonian

$H=-\frac{\hbar^{2}}{2 m_{1}} \nabla_{1}^{2}-\frac{\hbar^{2}}{2 m_{2}} \nabla_{2}^{2}+V_{1}\left(\mathbf{r}_{1}\right)+V_{2}\left(\mathbf{r}_{2}\right)+v\left(\left|\mathbf{r}_{1}-\mathbf{r}_{2}\right|\right)$,

where $V_{1}$ and $V_{2}$ are the confining potentials and $v(r)$ is the interaction between particles, represents a fundaample is the helium-atom problem that has played an important role in our understanding of electron correlation in atomic physics (see, e.g., Ref. [1]). Similarly, the problem of two atoms in a harmonic trap, which has attracted considerable recent attention (see, e.g., Refs. [2, [3, 4, , 5, [6, 7, , 8, 9, 10, 11, 12, 13]), is the key to our understanding of atomic correlation in a trapped manyatom quantum system. Such correlation differs qualitatively from the electron correlation because atoms attract each other at large distances and can form bound states.

Existing theories of two atoms in a trap have relied mostly upon the pseudopotential model of atomic interaction [14], and its generalizations [7]. While such models can work well in describing how the trap states, especially the lowest few, are affected by atomic interaction, they generally fail in describing how a molecular state is affected by trapping, with the only exception being the least bound molecular state with a very large scattering length. Furthermore, such theories do not adequately address nonzero partial waves, for which naive generalizations of the "shape-independent" approximation [2], using, e.g., the effect range theory (ERT) [15], would generally lead to incorrect results.

We present here a multiscale (2 length scales, to be exact) quantum-defect theory (QDT) for two identical atoms in a symmetric harmonic trap. It is a completely general theory that works for different partial waves, and mental class of problems in physics. One famous ex-

from deeply bound molecular states to highly excited trap states. In Sec. II] we expand our tool box of QDT for different long range potentials [1, 16, 17, 18, 19] by presenting a QDT for a symmetric harmonic potential. It is independently useful beyond the scope of two atoms in a trap. For example, it may be used to treat two nucleons outside of a closed shell [20].

In Sec. III this theory is combined with the angularmomentum-insensitive quantum-defect theory (AQDT) for the van der Waals interaction [21] to formulate a twoscale QDT that provides a systematic understanding of two identical atoms in a symmetric harmonic trap. Results and discussions are presented in Sec. IV including a discussion of the limitations of "shape-independent" approximations, and a universal spectrum for two atoms in a trap at the van der Waals length scale [21, 22, 23, 24. that shows, e.g., that a strong $p$ wave pairing can lead to a lower energy state around the threshold than a $s$ wave pairing. We will also show that two atoms in a trap has long-range correlation that becomes important for large scattering lengths, a result that has proven to be the key for generalizing the variational Monte Carlo (VMC) studies of few atoms in a trap to the regime of strong coupling [24]. Conclusions are given in Sec. V]

\section{QUANTUM-DEFECT THEORY FOR A SYMMETRIC HARMONIC POTENTIAL}

The goal of a QDT for a symmetric harmonic potential is to provide a systematic understanding to a class of problems described by the radial Schrödinger equation

$$
\left[-\frac{\hbar^{2}}{2 \mu} \frac{d^{2}}{d r^{2}}+\frac{\hbar^{2} l(l+1)}{2 \mu r^{2}}+V(r)-\epsilon\right] u_{\epsilon l}(r)=0,
$$

with

$$
V(r) \stackrel{r \rightarrow \infty}{\longrightarrow} \frac{1}{2} \mu \omega^{2} r^{2} .
$$

Unlike the standard textbook solution which requires

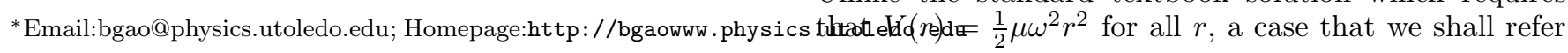


to as the "pure" harmonic oscillator, the QDT formulation is applicable to any $V(r)$ that is asymptotically a harmonic oscillator, but may differ from it at short distances in an arbitrary fashion.

As in any QDT formulation [1, 16, 17], we start by defining a pair of reference functions that are two linearly independent solutions for a symmetric harmonic potential

$$
\left[-\frac{\hbar^{2}}{2 \mu} \frac{d^{2}}{d r^{2}}+\frac{\hbar^{2} l(l+1)}{2 \mu r^{2}}+\frac{1}{2} \mu \omega^{2} r^{2}-\epsilon\right] v(r)=0 .
$$

The solutions can be easily found [25], and we will take

$$
\begin{aligned}
f_{e l}^{(h o)}(x)= & x^{l+1} e^{-x^{2} / 2} M\left(b, c, x^{2}\right), \\
g_{\text {el }}^{(h o)}(x)= & -\frac{2}{(2 l+1) \pi} x^{-l} e^{-x^{2} / 2} \\
& \times M\left(1+b-c, 2-c, x^{2}\right) .
\end{aligned}
$$

Here $e=\epsilon / \hbar \omega$ is a scaled energy. $x=r / \beta_{h o}$ is a radius scaled by $\beta_{h o}=(\hbar / \mu \omega)^{1 / 2}$, which is a length scale associated with the harmonic potential. $M$ is the confluent hypergeometric function [25], $b=(l+3 / 2-e) / 2$, and $c=l+3 / 2$. In this definition, $f_{e l}^{(h o)}$ is regular at the origin, and $g_{e l}^{(h o)}$ is irregular. They will be called the regular solution and the irregular solution, respectively. They are also chosen such that their Wronskian is given by

$$
W\left(f_{\epsilon_{s} l}^{(h o)}, g_{\epsilon_{s} l}^{(h o)}\right) \equiv f_{\epsilon_{s} l}^{(h o)} \frac{d g_{\epsilon_{s} l}^{(h o)}}{d x}-g_{\epsilon_{s} l}^{(h o)} \frac{d f_{\epsilon_{s} l}^{(h o)}}{d x}=\frac{2}{\pi} .
$$

With this definition of reference functions, the wave function $u_{\epsilon l}$ for any potential that is asymptotically a harmonic oscillator can be written, at sufficiently large distances, as

$$
u_{\epsilon l}(r)=A_{\epsilon l}\left[f_{e l}^{(h o)}(x)-K^{(h o)}(\epsilon, l) g_{e l}^{(h o)}(x)\right] .
$$

This defines the $K$ matrix, $K^{(h o)}$, for a symmetric harmonic potential, with its value being generally determined by matching Eq. (8) to the short-range solution.

Making use of the large $r$ asymptotic behaviors of $f^{(h o)}$ and $g^{(h o)}$, as given by [25]

$$
\begin{aligned}
f_{e l}^{(h o)} & \stackrel{r \rightarrow \infty}{\longrightarrow} \frac{\Gamma(c)}{\Gamma(b)} x^{-e-1 / 2} e^{+x^{2} / 2} \\
g_{\text {el }}^{(h o)} & \stackrel{r \rightarrow \infty}{\longrightarrow}-\frac{2}{(2 l+1) \pi}\left[\frac{\Gamma(2-c)}{\Gamma(1+b-c)} x^{-e-1 / 2} e^{+x^{2} / 2}\right. \\
& \left.-\frac{1}{\pi} \sin (\pi c) \Gamma(b) \Gamma(2-c) x^{+e-1 / 2} e^{-x^{2} / 2}\right]
\end{aligned}
$$

and enforcing the boundary condition, $u_{\epsilon l}(r) \rightarrow 0$, at infinity, we obtain the following equation that gives the the energy spectrum of Eq. (2) as the crossings points of two functions:

$$
\chi_{l}^{(h o)}(e)=K^{(h o)}(\epsilon, l) .
$$

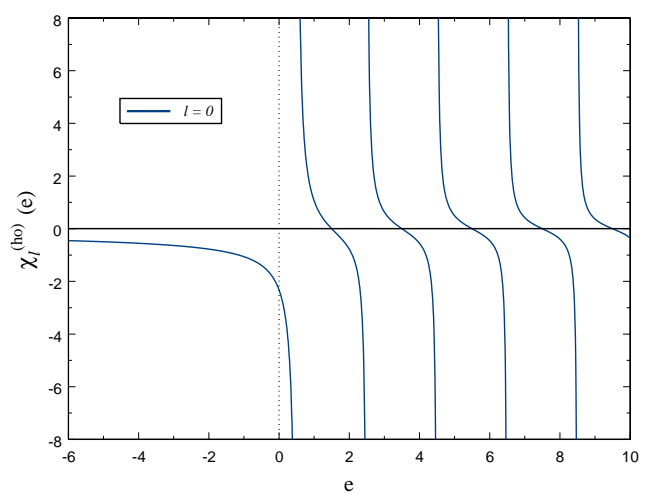

FIG. 1: The $\chi_{l}^{(h o)}(e)$ function for the $s$ wave. The energy spectrum for any potential that is asymptotically a symmetric harmonic oscillator is given by the crossing points of this function with a short-range $K$ matrix $K^{(h o)}(\epsilon, l)$ defined by Eq. (8). The special case of $K^{(h o)}(\epsilon, l)=0$ for all energies corresponds to a pure harmonic oscillator.

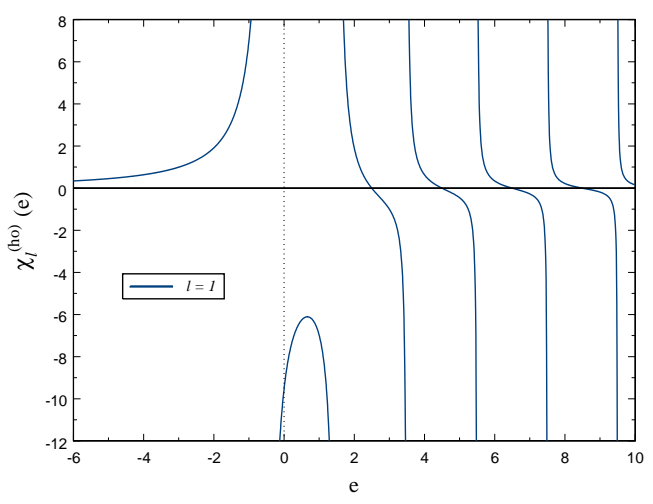

FIG. 2: The same as Figure 1 except that it is for the $p$ wave.

Here

$$
\chi_{l}^{(h o)}(e)=(-1)^{l+1}[\Gamma(l+3 / 2)]^{2} \frac{\Gamma[1-(e+l+3 / 2) / 2]}{\Gamma[(-e+l+3 / 2) / 2]},
$$

is a universal function of the scaled energy $e$ that is determined solely by the harmonic potential, and $K^{(h o)}$ is the $K$ matrix that encapsulates all the short-range physics. Plots of $\chi_{l}^{(h o)}(e)$ for the $s$ and $p$ waves are shown in Figs. 1 and 2 respectively.

Equations (11) and (12) give a rigorous formulation of energy spectrum for any potential that is asymptotically a harmonic oscillator. It can be implemented numerically, or used as a basis for approximate analytic solutions. The case of a pure symmetric harmonic oscillator is included as a special case, corresponding to 
$K^{(h o)}(\epsilon, l)=0$ for all $\epsilon$, with a well-known energy spectrum of $\epsilon=\hbar \omega(2 j+l+3 / 2)$, where $j=0,1,2, \ldots$.

From Eq. (11), it is clear that the key to finding the energy spectrum is to find the $K$ matrix $K^{(h o)}$, as $\chi_{l}^{(h o)}$ is already known analytically. For this purpose, we note that the reference functions $f^{(h o)}$ and $g^{(h o)}$ have the following small $r$ asymptotic behaviors that can be derived from a proper expansion of the confluent hypergeometric function [25]. For $\epsilon>0$, we have

$$
\begin{aligned}
& f_{\text {el }}^{(h o)} \stackrel{r \ll \beta_{h o}}{\longrightarrow} \sqrt{\frac{2}{\pi}} \frac{2^{l+1 / 2} \Gamma(l+3 / 2)}{\left(k \beta_{h o}\right)^{l+1}}(k r) j_{l}(k r), \\
& g_{\text {el }}^{(h o)} \stackrel{r \ll \beta_{h o}}{\longrightarrow} \sqrt{\frac{2}{\pi}} \frac{\left(k \beta_{h o}\right)^{l}}{2^{l+1 / 2} \Gamma(l+3 / 2)}(k r) y_{l}(k r),
\end{aligned}
$$

where $k=\left(2 \mu \epsilon / \hbar^{2}\right)^{1 / 2}$. For $\epsilon<0$, we have

$$
\begin{aligned}
& f_{\text {el }}^{(h o)} \stackrel{r \ll \beta_{h_{0}}}{\longrightarrow} \frac{2^{l+1 / 2} \Gamma(l+3 / 2)}{\left(\kappa \beta_{h o}\right)^{l+1}}(\kappa r)^{1 / 2} I_{l+1 / 2}(\kappa r),(15) \\
& g_{\text {el }}^{(h o)} \stackrel{r \ll \beta_{h_{o}}}{\longrightarrow} \frac{(-1)^{l+1}\left(\kappa \beta_{h o}\right)^{l}}{2^{l+1 / 2} \Gamma(l+3 / 2)}(\kappa r)^{1 / 2} I_{-l-1 / 2}(\kappa r)
\end{aligned}
$$

where $\kappa=\left(-2 \mu \epsilon / \hbar^{2}\right)^{1 / 2}$. For interactions $V(r)$ that deviate from the harmonic potential only in the region of $r \ll \beta_{h o}$, these behaviors greatly facilitate the matching to the short-range solution, from which $K^{(h o)}$ can be determined. This is illustrated in our treatment of two identical atoms in a symmetric harmonic trap, to be presented in the next section.

\section{TWO IDENTICAL ATOMS IN A SYMMETRIC HARMONIC TRAP}

Two interacting atoms in symmetric harmonic trap are described by a Hamiltonian

$$
\begin{aligned}
H= & -\frac{\hbar^{2}}{2 m_{1}} \nabla_{1}^{2}-\frac{\hbar^{2}}{2 m_{2}} \nabla_{2}^{2}+\frac{1}{2} m_{1} \omega_{1}^{2} r_{1}^{2}+\frac{1}{2} m_{2} \omega_{2}^{2} r_{2}^{2} \\
& +v\left(\left|\mathbf{r}_{1}-\mathbf{r}_{2}\right|\right),
\end{aligned}
$$

where $v(r)$ represents the interaction between them. To be specific, we restrict ourselves here to a class of problems for which the atomic interaction is characterized, at large distances, by an attractive $1 / r^{6}$ van der Waals potential

$$
v(r) \stackrel{r \rightarrow \infty}{\longrightarrow}-C_{6} / r^{6}
$$

which has an associated length scale of $\beta_{6}=$ $\left(2 \mu C_{6} / \hbar^{2}\right)^{1 / 4}$ [18].

For two atoms having the same trapping frequencies, namely $\omega_{1}=\omega_{2}=\omega$, which include of course the case of two identical atoms of interest here, the center-of-mass motion and the relative motion are separable, and the solution of two identical atoms in a symmetric harmonic trap reduces to the solution of Eq. (2) with

$$
\begin{aligned}
V(r) & =v(r)+\frac{1}{2} \mu \omega^{2} r^{2} \\
& =-C_{6} / r^{6}+\frac{1}{2} \mu \omega^{2} r^{2}, r \geq r_{0},
\end{aligned}
$$

where $r_{0}$ represents the radius inside which the interactions of shorter range than $\beta_{6}$, such as the $-C_{8} / r^{8}$ correction, would come into play.

Since the $V(r)$ characterized by Eq. (19) is asymptotically a harmonic oscillator, it is amenable for the QDT treatment of Sec. II In particular, the solution for the energy spectrum reduces to finding the $K$ matrix $K^{(h o)}$ for the class of problems defined by Eqs. (18) and (19). This will be accomplished here by taking advantage of the disparate length scales in the system.

For $r \geq r_{0}$, the potential $V(r)$ as given by Eq. (19) has two length scales. In addition to $\beta_{6}$ that is associated with the van der Waals interaction, the harmonic trapping potential has a length scale that can be taken either as the $\beta_{h o}$ defined earlier, or as $a_{h o}=(\hbar / m \omega)^{1 / 2}=$ $\beta_{h o} / \sqrt{2}$. We will use both interchangeably, but will emphasize $a_{h o}$ for the sake of easier comparison with other results.

For atoms in a typical magnetic or optical trap, $a_{h o}$ is of the order of a micron, which is much greater than $\beta_{6}$ that is of the order of $100 \mathrm{au}$ or about $50 \mathrm{~nm}$. Under this condition of $\beta_{6} \ll a_{h o}$, which we will call the limit of weak confinement, the van der Waals and the trapping potentials operate on distinctive lengths scales. In the region of $r \gg \beta_{6}$, the van der Waals interaction is negligible, and we have

$$
u_{I I}(r)=A\left[f_{e l}^{(h o)}-K^{(h o)}(\epsilon, l) g_{e l}^{(h o)}\right] .
$$

In the region of $r_{0} \leq r \ll a_{h o}$, the harmonic potential is negligible, and the interaction is dominated by the van der Waals interaction. Here the wave function can be written as 21]

$$
u_{I}(r)=B\left[f_{\epsilon_{s} l}^{c(6)}-K^{c}(\epsilon, l) g_{\epsilon_{s} l}^{c(6)}\right] .
$$

where $f_{\epsilon_{s} l}^{c(6)}$ and $g_{\epsilon_{s} l}^{c(6)}$ are the reference functions for the van der Waals potential, and $K^{c}$ is the corresponding short-range $K$ matrix [18, 21, 22].

For weak confinement defined by $\beta_{6} \ll a_{h o}$, there exists an intermediate region $\beta_{6} \ll r \ll a_{h o}$ in which either, or both, the van der Waals potential and the trapping potential can be ignored. In this region, the wave function can be written either in the form of the inner solution as given by Eq. (21), or in the form of the outer solution as given by Eq. (20), and they must agree with each other.

Since $r / a_{h o} \ll 1$ in the intermediate region, $f_{e l}^{(h o)}$ and $g_{e l}^{(h o)}$ are given by Eqs. (13)-(16). In the same region, $r / \beta_{6} \gg 1$ and the reference functions for the van der Waals potential are given for $\epsilon \geq 0$ by [18, 26]

$$
f_{\epsilon_{s} l}^{c(6)}=\sqrt{\frac{2}{\pi k \beta_{6}}}(k r)\left[Z_{f f}^{c(6)} j_{l}(k r)+Z_{f g}^{c(6)} y_{l}(k r)\right],
$$




$$
g_{\epsilon_{s} l}^{c(6)}=\sqrt{\frac{2}{\pi k \beta_{6}}}(k r)\left[Z_{g f}^{c(6)} j_{l}(k r)+Z_{g g}^{c(6)} y_{l}(k r)\right],
$$

and for $\epsilon<0$ by $[18,26$ ]

$$
\begin{aligned}
f_{\epsilon_{s} l}^{c(6)} & =\frac{1}{2}\left[W_{f-}^{c(6)}+2(-1)^{l} W_{f+}^{c(6)}\right]\left(r / \beta_{6}\right)^{1 / 2} I_{l+1 / 2}(\kappa r) \\
& +\frac{1}{2}\left[W_{f-}^{c(6)}-2(-1)^{l} W_{f+}^{c(6)}\right]\left(r / \beta_{6}\right)^{1 / 2} I_{-l-1 / 2}(\kappa(24) \\
g_{\epsilon_{s} l}^{c(6)} & =\frac{1}{2}\left[W_{g-}^{c(6)}+2(-1)^{l} W_{g+}^{c(6)}\right]\left(r / \beta_{6}\right)^{1 / 2} I_{l+1 / 2}(\kappa r) \\
& +\frac{1}{2}\left[W_{g-}^{c(6)}-2(-1)^{l} W_{g+}^{c(6)}\right]\left(r / \beta_{6}\right)^{1 / 2} I_{-l-1 / 2}(\kappa(25)
\end{aligned}
$$

Here the $Z^{c(6)}$ and $W^{c(6)}$ matrices describe the propagation of a wave function in a $-C_{6} / r^{6}$ type of potential from small to large distances, and vice versa [18, 21, 27]. Their elements of are all universal functions of a scaled energy $\epsilon_{s}=\epsilon / s_{E}$, where $s_{E}=\left(\hbar^{2} / 2 \mu\right)\left(1 / \beta_{6}\right)^{2}$ is the energy scale associated with the van der Waals interaction. Explicit expressions for the elements of the $Z^{c(6)}$ can be found in Ref. 27]. The $W^{c(6)}$ matrix, which is related to the $W$ matrix defined in Ref. [18] by a linear transformation [28], is given by

$$
\begin{aligned}
W_{f-}^{c(6)}\left(\epsilon_{s}\right) & =\frac{2^{-1 / 2} G_{\epsilon l}(\nu)}{\left(X_{\epsilon l}^{2}+Y_{\epsilon l}^{2}\right) \sin \pi \nu}\left[\left(1+M_{\epsilon l}\right) \sin (\pi \nu / 2) X_{\epsilon l}+\left(1-M_{\epsilon l}\right) \cos (\pi \nu / 2) Y_{\epsilon l}\right], \\
W_{f+}^{c(6)}\left(\epsilon_{s}\right) & =\frac{2^{-1 / 2} G_{\epsilon l}(\nu) \cos \pi \nu}{\left(X_{\epsilon l}^{2}+Y_{\epsilon l}^{2}\right)}\left[\left(1-M_{\epsilon l}\right) \sin (\pi \nu / 2) X_{\epsilon l}+\left(1+M_{\epsilon l}\right) \cos (\pi \nu / 2) Y_{\epsilon l}\right], \\
W_{g-}^{c(6)}\left(\epsilon_{s}\right) & =\frac{2^{-1 / 2} G_{\epsilon l}(\nu)}{\left(X_{\epsilon l}^{2}+Y_{\epsilon l}^{2}\right) \sin \pi \nu}\left[\left(1-M_{\epsilon l}\right) \cos (\pi \nu / 2) X_{\epsilon l}-\left(1+M_{\epsilon l}\right) \sin (\pi \nu / 2) Y_{\epsilon l}\right], \\
W_{g+}^{c(6)}\left(\epsilon_{s}\right) & =\frac{2^{-1 / 2} G_{\epsilon l}(\nu) \cos \pi \nu}{\left(X_{\epsilon l}^{2}+Y_{\epsilon l}^{2}\right)}\left[\left(1+M_{\epsilon l}\right) \cos (\pi \nu / 2) X_{\epsilon l}-\left(1-M_{\epsilon l}\right) \sin (\pi \nu / 2) Y_{\epsilon l}\right] .
\end{aligned}
$$

Here $M_{\epsilon l}=G_{\epsilon l}(-\nu) / G_{\epsilon l}(\nu)$, with $\nu, X_{\epsilon l}, Y_{\epsilon l}$, and $G_{\epsilon l}$, all of which are functions of the scaled energy $\epsilon_{s}$, being defined in Ref. [18].

Comparing, in the intermediate region, the inner solution given by Eqs. (21) and (22)-(25) with the outer solution given by Eqs. (20) and (13)-(16), we obtain, for $\epsilon>0$,

$$
K^{(h o)}=[\Gamma(l+3 / 2)]^{2} \frac{\tan \delta_{l}}{(e / 2)^{l+1 / 2}},
$$

where

$$
\tan \delta_{l}(\epsilon)=-\left(Z_{f g}^{c(6)}-Z_{g g}^{c(6)} K^{c}\right)\left(Z_{f f}^{c(6)}-Z_{g f}^{c(6)} K^{c}\right)^{-1},
$$

is the physical $\mathrm{K}$ matrix for atomic scattering in free space as given in AQDT [21]. For $\epsilon<0$, we obtain

$$
K^{(h o)}=(-1)^{l} \frac{[\Gamma(l+3 / 2)]^{2}}{(|e| / 2)^{l+1 / 2}}\left\{\frac{\chi_{l}^{c(6)}-K^{c}-2(-1)^{l}\left[\left(W_{f+}^{c(6)} / W_{g-}^{c(6)}\right)-K^{c}\left(W_{g+}^{c(6)} / W_{g-}^{c(6)}\right)\right]}{\chi_{l}^{c(6)}-K^{c}+2(-1)^{l}\left[\left(W_{f+}^{c(6)} / W_{g-}^{c(6)}\right)-K^{c}\left(W_{g+}^{c(6)} / W_{g-}^{c(6)}\right)\right]}\right\}
$$

where $\chi_{l}^{c(6)}\left(\epsilon_{s}\right)=W_{f-}^{c(6)} / W_{g-}^{c(6)}$ is the $\chi$-function that determines the molecular spectrum in the absence of trapping [21].

Equations (11)-(12) and (30)-(32) give a complete description of the energy spectrum for two idential atoms in a symmetric harmonic trap, from deeply-bound molecular states to highly excited trap states. The only assumption in the theory is the assumption of weak confinement as specified by $\beta_{6} / a_{h o} \ll 1$, which is well satisfied under all existing experimental conditions.
Other than the two energy scaling parameters $\hbar \omega$ and $s_{E}$, the only parameter in the theory is $K^{c}(\epsilon, l)$, which characterizes the interactions of shorter range than $\beta_{6}$. It can be replaced by other equivalent short-range parameters such as the quantum-defect $\mu^{c}(\epsilon, l)$ 29] or the $K_{l}^{0}(\epsilon, l)$ parameter [30, 31]. 


\section{RESULTS AND DISCUSSIONS}

\section{A. Universal spectrum at the length scale of $\beta_{6}$}

The results given by Eqs. (11), (12), (30)-(32) can be written in different forms that are convenient for different purposes. For conceptual understanding, it is best to rearrange them so that the entire energy spectrum is given by the solutions of a single equation

$$
\chi_{l}^{(h o, 6)}\left(e, \beta_{6} / a_{h o}\right)=K^{c}(\epsilon, l),
$$

where

$$
\chi_{l}^{(h o, 6)}=\frac{Z_{f g}^{c(6)}\left(\epsilon_{s}\right)-Z_{f f}^{c(6)}\left(\epsilon_{s}\right) \xi_{l}^{(h o)}(e)}{Z_{g g}^{c(6)}\left(\epsilon_{s}\right)-Z_{g f}^{c(6)}\left(\epsilon_{s}\right) \xi_{l}^{(h o)}(e)}
$$

for $e>0$,

$$
\chi_{l}^{(h o, 6)}=\frac{\chi_{l}^{c(6)}\left(\epsilon_{s}\right)-\alpha_{l}^{(h o)}(e)\left[W_{f+}^{c(6)}\left(\epsilon_{s}\right) / W_{g-}^{c(6)}\left(\epsilon_{s}\right)\right]}{1-\alpha_{l}^{(h o)}(e)\left[W_{g+}^{c(6)}\left(\epsilon_{s}\right) / W_{g-}^{c(6)}\left(\epsilon_{s}\right)\right]},
$$

for $e<0$, and we have defined

$$
\xi_{l}^{(h o)}(e)=(-1)^{l}(e / 2)^{l}(|e| / 2)^{1 / 2} \frac{\Gamma[1-(e+l+3 / 2) / 2]}{\Gamma[(-e+l+3 / 2) / 2]},
$$

and

$$
\alpha_{l}^{(h o)}(e)=2(-1)^{l} \frac{1-\xi_{l}^{(h o)}(e)}{1+\xi_{l}^{(h o)}(e)} .
$$

The $\chi_{l}^{(h o, 6)}$ function is a universal function that is applicable to any two identical atoms in a symmetric harmonic trap, provided they interact via the $-C_{6} / r^{6}$ type of van der Waals potential at large interatomic separations. The strengths of interactions, as characterized by $C_{6}$ and $\omega$, play a role only through energy scaling parameters $s_{E}$ and $\hbar \omega$. Specifically, the $\chi_{l}^{(h o, 6)}$ function is made up terms that depend on energy through two different scaled energies, $\epsilon_{s}=\epsilon / s_{E}$ and $e=\epsilon / \hbar \omega$ that are related by $\epsilon_{s}=\left(\beta_{6} / a_{h o}\right)^{2} e$. In other words, it is made up functions that vary on two distinctive energy scales: the $\xi^{(h o)}$ and the $\alpha^{(h o)}$ functions that varies on the scale of $\hbar \omega$, and the $Z^{c(6)}$ and $W^{c(6)}$ matrix elements that vary on a scale of $s_{E}$.

The solutions, more precisely the inverse, of Eq. (33) can be written as

$$
e=\widetilde{\Omega}_{i}^{(l)}\left(K^{c}, \beta_{6} / a_{h o}\right),
$$

where, similar to $\chi_{l}^{(h o, 6)}$, the $\widetilde{\Omega}_{i}^{(l)}$ are universal functions that are uniquely determined by the exponent of the van der Waals interaction $(n=6)$, and the exponent of the trapping potential ( 2 for the harmonic trap). Instead of the parameter $K^{c}$, the same universal functions can also be expressed in terms of equivalent parameters such as the quantum-defect $\mu^{c}$ [29], or the $K_{l}^{0}$ parameter [30], all of which are well defined for all $l$.

When the energy and angular momentum dependence of the short-range parameter $K^{c}$ is included, Eqs. (33) and (38) are exact, and applicable to arbitrary energy and partial waves. Ignoring the energy and the $l$ dependence of $K^{c}$, which in the case of a single channel is due entirely to interactions of shorter range than $\beta_{6}$ [21, 29], the solutions of Eq. (33), namely Eq. (38) with $K^{c}=K^{c}(\epsilon=0, l=0)$, or its variations, give what we call the universal spectrum at length scale $\beta_{6}[21,22,24]$. It is followed by all two-atom systems in a trap with $-C_{6} / r^{6}$ type of long-range interaction, over a range of energies that is hundreds of $s_{E}$ around the threshold [21, 28], which far exceeds all energies of interest in cold-atom physics. (As an example, $s_{E}=9.331 \times 10^{-4} \mathrm{~K}$ for ${ }^{23} \mathrm{Na}$.) Other than the energy scaling parameter $s_{E}$ that is determined by the $C_{6}$ coefficient and the atomic mass, all energy levels in this energy range, including states of different $l$, are determined, in the case of single channel, by two parameters, $K^{c}=K^{c}(\epsilon=0, l=0)$ and $\beta_{6} / a_{h o}$. This is an example of universal spectrum at the second longest length scale in the system [24], as opposed to the universal spectrum at the longest length scale, which would have required only a single parameter [21].

Depending on the physics of interest, the universal spectrum at length scale $\beta_{6}$ can also be expressed in terms of other parameters. In particular, it can be expressed in terms of the $s$ wave scattering length, $a_{0}$, as

$$
e=\Omega_{i}^{(l)}\left(a_{0} / a_{h o}, \beta_{6} / a_{h o}\right),
$$

since $a_{0}$ can be related to $K^{c}=K^{c}(\epsilon=0, l=0)$ by

$$
a_{0} / \beta_{n}=\left[b^{2 b} \frac{\Gamma(1-b)}{\Gamma(1+b)}\right] \frac{K^{c}(0,0)+\tan (\pi b / 2)}{K^{c}(0,0)-\tan (\pi b / 2)},
$$

where $b=1 /(n-2)$ with $n=6$ [29, 32]. Similar representations of universal spectrum can also be defined more generally for $N$ atoms $(N>2)$ in a trap [22, 23, 24].

Figure 3 illustrates the universal $s$ wave spectrum at length scale $\beta_{6}$ for two identical atoms in a symmetric harmonic trap. It uses the representation of Eq. (39) to facilitate comparison with the shape-independent approximation (see Ref. [2] and Sec. IVB]). The $\Omega^{(l=0)}$ functions of two variables $a_{0} / a_{h o}$ and $\beta_{6} / a_{h o}$ are plotted here as functions of $a_{0} / a_{h o}$ for different values of $\beta_{6} / a_{h o}$. In the small range of energies shown in the figure, which corresponds to $\epsilon \sim \hbar \omega \ll s_{E}$, the universal spectrum approaches that of the shape-independent results [2] in the limit of $\beta_{6} / a_{h o} \rightarrow 0$ [24].

Figures 4 and 5 both illustrate the universal $p$ wave spectrum at length scale $\beta_{6}$. They also serve to illustrate how different representations of the universal spectrum can serve different purposes in terms of physical understanding. In Fig. 4, the universal $p$ wave spectrum is plotted as a function of $a_{0} / \beta_{6}$ for different values of $\beta_{6} / a_{h o}$. It gives the best illustration that, in the case of single channel, the $s$ wave scattering length determines 


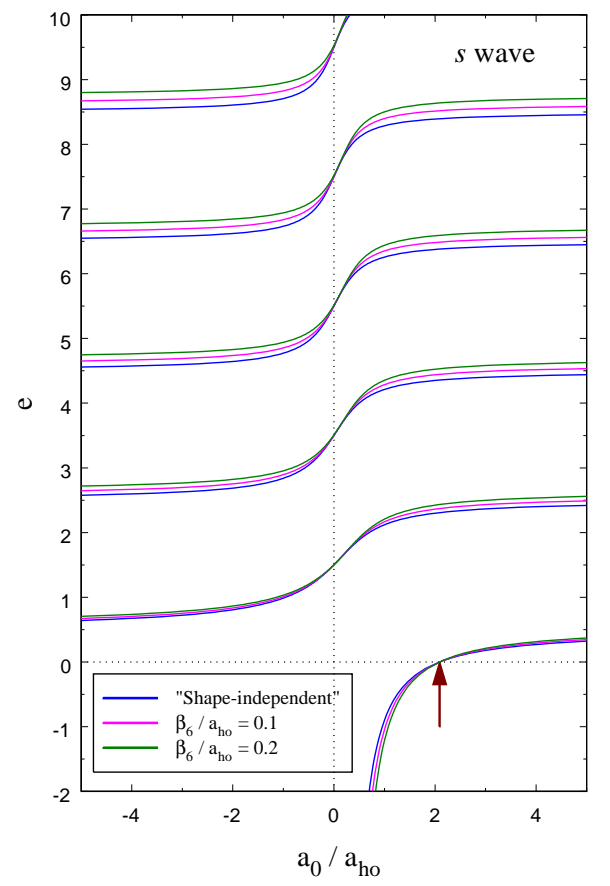

FIG. 3: Universal $s$ wave spectrum at length scale $\beta_{6}$ for two identical atoms in a symmetric harmonic trap and with an asymptotic interaction of the type of $-1 / r^{6}$. The arrow points to the $s$ wave scattering length, $a_{0 x}$, beyond which the least-bound molecular $s$ state is pushed to positive energies.

not only the $s$ wave spectrum, but also the spectra of other partial waves including the $p$ wave 21]. It also determines the $p$ wave scattering length $a_{1}$ (also called scattering volume as it has the dimension of a volume) through [31]

$$
a_{1} / \beta_{6}^{3}=\frac{[\Gamma(1 / 4)]^{2}}{18 \pi} \frac{a_{0}-\bar{a}_{0}}{2 \bar{a}_{0}-a_{0}},
$$

where $\bar{a}_{0}=2 \pi \beta_{6} /[\Gamma(1 / 4)]^{2}=0.4779888 \beta_{6}$ is the mean $s$ wave scattering length of Gribakin and Flambaum 33]. Figure 4 shows that the $p$ wave spectrum, in the case of single channel, is strongly influenced by the atomic interaction only when the $s$ wave scattering length is around $2 \bar{a}_{0}=0.955978 \beta_{6}$, which corresponds to having a $p$ wave bound state right at the threshold [27, 29]. For $a_{0}$ slightly larger than $2 \bar{a}_{0}, a_{1}$ is large and negative [see Eq. (41)], the lowest few trap states are strongly affected by a $p$ wave shape resonance near the threshold [30]. For $a_{0}$ slightly less than $2 \bar{a}_{0}, a_{1}$ is large and positive, and there is a $p$ wave molecular state close to the threshold [31].

In Fig. 5, the universal $p$ wave spectrum is plotted as a function of $a_{1} / a_{h o}^{3}$ for different values of $\beta_{6} / a_{h o}$. The advantages of this representation are twofold. First, it facilitates comparison with the $p$ wave shape-independent approximation to be discussed in Sec. IVB Second,

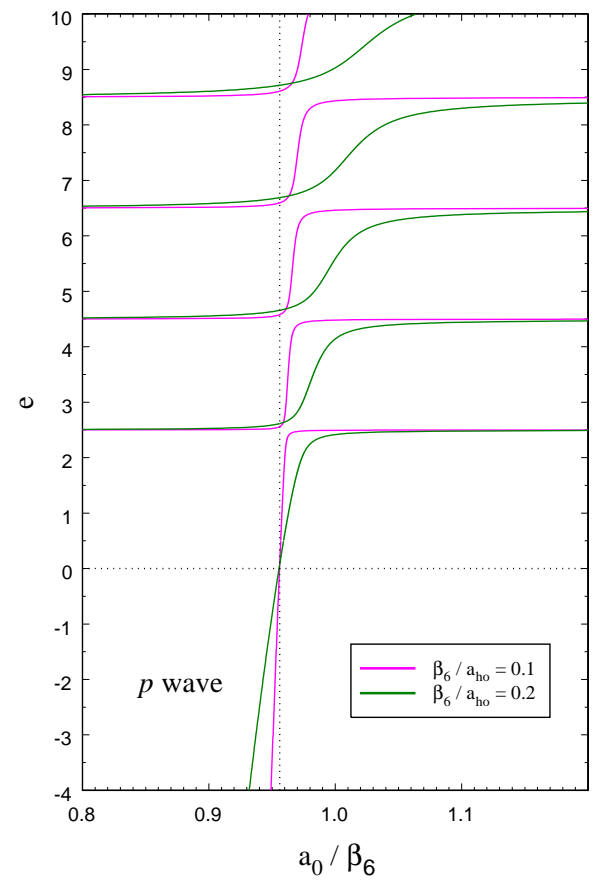

FIG. 4: Universal $p$ wave spectrum at length scale $\beta_{6}$ for two identical atoms in a symmetric harmonic trap and with an asymptotic interaction of the type of $-1 / r^{6}$, plotted here versus a scaled $s$ wave scattering length, $a_{0} / \beta_{6}$.

while the representation shown in Fig. 4 is applicable only in the case of single channel, the representation shown in Fig. 5 would apply even in multichannel cases, provided the energy dependence of $K^{c}$ due to closed channels is negligible in energy range of interest [26, 28]. This is because in representing the $p$ wave spectrum as a function of $a_{1}$, instead of $a_{0}$, one is only making use of the energy-independence of $K^{c}$, not its angularindependence, which takes on different characteristics (still related) for multichannel problems 26, 28]. Specifically, this representation is obtain from solving Eq. (33) using $K^{c}=K^{c}(\epsilon=0, l=1)$, which is related to the $a_{1}$ through the following relations [30, 31]

$$
a_{1}=-\bar{a}_{1}\left(1+\frac{1}{K_{l=1}^{0}(\epsilon=0)}\right),
$$

where $\bar{a}_{1}=[\Gamma(1 / 4)]^{2} \beta_{6}^{3} /(36 \pi)$ is a mean $p$ wave scattering length, and

$$
K_{l}^{0}(\epsilon=0)=\frac{c_{l}-K^{c}(\epsilon=0, l)}{1+c_{l} K^{c}(\epsilon=0, l)}
$$

where $c_{l}=\tan \left(\frac{1}{4} l \pi+\frac{1}{8} \pi\right)$.

Comparing either Fig. 4 or 5 with Fig. 3 leads to one of the more important conclusions of this work. That is, unlike the noninteracting particles in a trap for which the 


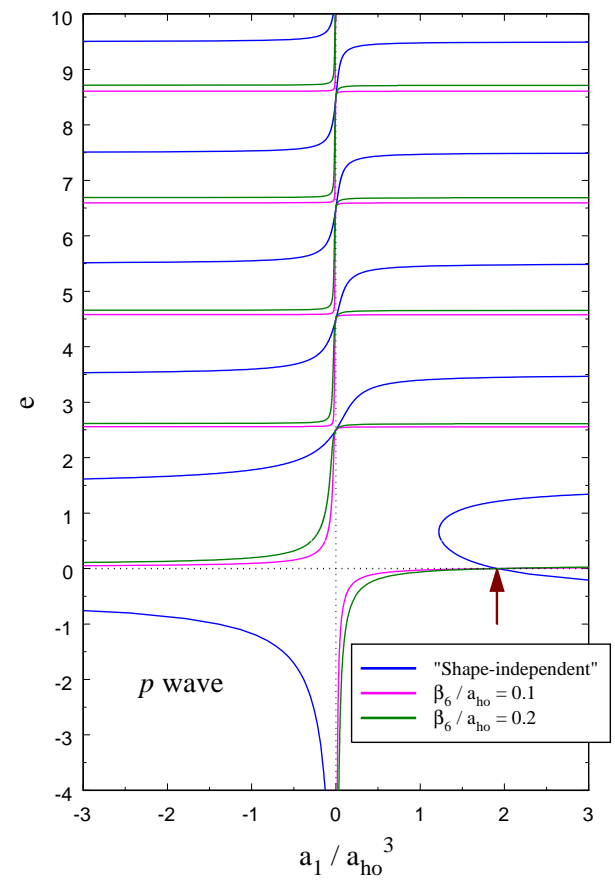

FIG. 5: Universal $p$ wave spectrum at length scale $\beta_{6}$ for two identical atoms in a symmetric harmonic trap and with an asymptotic interaction of the type of $-1 / r^{6}$, plotted here versus a scaled $p$ wave scattering length (volume) $a_{1} / a_{h o}^{3}$. The arrow points to the $p$ wave scattering length, $a_{1 x}$, beyond which the least-bound molecular $p$ state is pushed to positive energies.

lowest $p$ state energy is always greater than that of the $s$ states, a strong $p$ wave pairing $\left(a_{1} \sim a_{h o}^{3}\right.$ or greater $)$ for interacting particles can lead to a lower energy state around the threshold than a $s$ wave pairing. This state is, for large and negative $a_{1}$, a $p$ wave shape resonance stabilized by the trap, and is a $p$ wave molecular state for large and positive $a_{1}$. Similar statements can also be made for other, higher partial waves. Furthermore, we expect the same physics to persist in many-atom systems, which will be a subject of future investigations. Other characteristics of the universal spectra are addressed separately in subsequent sections.

\section{B. Limitations of "shape-independent" approximations}

All previous results on two atoms in a symmetric harmonic trap can be easily derived as various approximations within our theory. In particular, the "shapeindependent" approximation corresponds to ignoring energy dependence of $K^{(h o)}$, and taking it to be its value

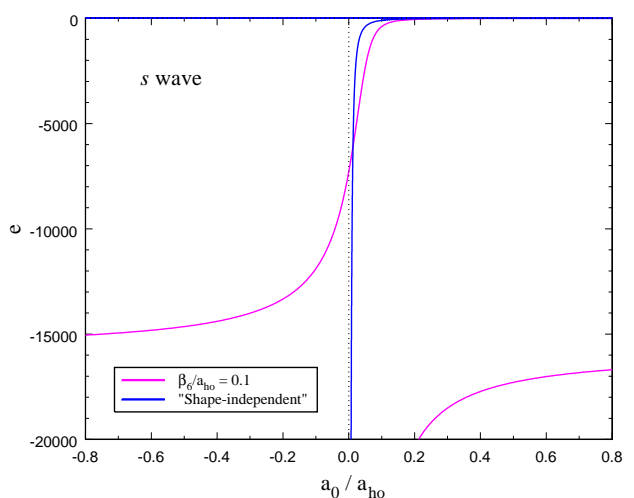

FIG. 6: Universal $s$ state spectrum at length scale $\beta_{6}$ for two identical atoms in a symmetric harmonic trap and with an asymptotic interaction of the type of $-1 / r^{6}$, plotted to illustrate the failure of the shape-independent approximation away from the threshold.

at zero energy, namely,

$$
K^{(h o)} \approx-\frac{[\Gamma(l+3 / 2)]^{2}}{\left(\beta_{h o} / 2\right)^{2 l+1}} \lim _{k \rightarrow 0}\left[-\frac{\tan \delta_{l}}{k^{2 l+1}}\right] .
$$

For partial waves with well-defined scattering lengths, $a_{l}=\lim _{k \rightarrow 0}\left[-\tan \delta_{l} / k^{2 l+1}\right]$, this approximation used in Eq. (11) leads to the following equation for the energy spectrum:

$$
(-1)^{l}\left(\frac{1}{2}\right)^{l+1 / 2} \frac{\Gamma[1-(e+l+3 / 2) / 2]}{\Gamma[(-e+l+3 / 2) / 2]}=a_{l} / a_{h o}^{2 l+1},
$$

for both positive and negative energies. For $l=0$, Eq. (45) reproduces the result of Ref. [2], derived using a delta-function pseudopotential [14].

Figure 3 has shown that for the $s$ wave, the shapeindependent approximation gives a good approximation to the universal spectrum under weak confinement $\left(\beta_{6} / a_{h o} \ll 1\right)$ and for energies $|\epsilon| \sim \hbar \omega \ll s_{E}$. The shape dependence is more important for strong coupling $\left(a_{0} \sim a_{h o}\right.$ or greater), but especially for energies further away from the threshold. In particular, the shapeindependent approximation breaks down for all energies $\epsilon \sim s_{E}$ or greater, for which the energy dependence of $K^{(h o)}$ due to the long-range van der Waals interaction can no longer be ignored [26, 30]. For example, it does not give the proper molecular binding energy for small positive scattering lengths, and it fails completely to describe molecular states of negative scattering length, as illustrated in Fig. 6. In reality, these more deeply bound molecular states approach those of a free molecule in the absence of a trap, with the trapping potential serving as a weak perturbation, as to be discussed further in Sec. IVD

With the relative success of the "shape-independent" approximation for the $s$ wave in the threshold region, it 
is important to emphasize its severe limitations for any partial waves other than the $s$ wave. For atoms with $-C_{6} / r^{6}$ type of van der Waals interaction, the "shapeindependent" approximation clearly fails for $l \geq 2$, for which there are no well-defined scattering lengths [30, 34]. Even for the $p$ wave, it is applicable only at zero energy, or for weak $p$ wave coupling as characterized by $a_{1} \sim$ $\beta_{6}^{3} \ll a_{h o}^{3}$ or smaller, as illustrated in Fig. 4. This failure of the "shape-independent" approximation for $l>0$ is directly related to the failure of the effective range theory (ERT) [15] in describing the shape resonances close to the threshold, and more generally to the failure of ERT in describing Feshbach resonances in nonzero partial waves [26].

Our theory allows for simple generalizations beyond the shape-independent approximation. For example, for positive energies, Eqs. (11), (12) and (30) that determine the energy spectrum can be rewritten as

$$
-\xi_{l}^{(h o)}(e)=\tan \delta_{l}(\epsilon) .
$$

Instead of the effective-range expansion for $\tan \delta_{l}$, which leads to the "shape-independent" approximation, one can simply use the corresponding QDT expansion for $-C_{6} / r^{6}$ type of potential [30]. The results would be applicable from zero energy up to $\epsilon \sim s_{E}$ for the $s$ wave, and over a greater range of energies for higher partial waves. This energy range, while much smaller than that described by the universal spectrum Eq. (33), already exceeds the range of interest in existing experiments [12].

\section{Trap states and molecular states}

The states of two atoms in a trap can be classified into trap states and molecular states. The former corresponds to states that evolve into diatomic continuum as the trap is turned off adiabatically $\left(a_{h o} \rightarrow \infty\right)$. The latter corresponds to states that evolve into bound molecular states in the same limit. Note that the molecular states would not have existed in the hard-sphere atomic model [6].

The molecular state of highest energy correspond to the ones in Figs. 3 3 that cross the zero energy. It is the least-bound molecular state that gets pushed up in energy by the trapping potential. The crossing into positive energy can happen either by tuning up the scattering length, which has the effect of making the binding energy of the molecule sufficiently small in the absence of the trap, or by tuning up the trap frequency.

The crossing points, which correspond to having a state right at $\epsilon=0$, can be easily found through Eq. (45), which is exact at zero energy for partial waves with well defined scattering lengths. For $s$ and $p$ waves, it gives

$$
a_{l x}=(-1)^{l}\left(\frac{1}{2}\right)^{l+1 / 2} \frac{\Gamma(1 / 4-l / 2)}{\Gamma(l / 2+3 / 4)} a_{h o}^{2 l+1} .
$$

For the $s$ wave with a fixed trap frequency, it gives

$$
a_{0 x}=\frac{[\Gamma(1 / 4)]^{2}}{2 \pi} a_{h o}=2.0920992 a_{h o},
$$

beyond which the least bound molecular states is pushed to positive energy. For a fixed $a_{0} \gg \beta_{6}$, the same equation determines the crossing trap frequency

$$
\omega_{0 x}=\frac{[\Gamma(1 / 4)]^{4}}{(2 \pi)^{2}} \frac{\hbar}{m a_{0}^{2}},
$$

beyond which the least bound $s$ wave molecular state is pushed into positive energy.

For the $p$ wave, it gives

$$
a_{1 x}=\frac{8 \pi}{[\Gamma(1 / 4)]^{2}} a_{h o}^{3}=1.9119552 a_{h o}^{3} .
$$

For a fixed $a_{1} \gg \beta_{6}^{3}$, it determines the crossing trap frequency

$$
\omega_{1 x}=\frac{4 \pi^{2 / 3}}{[\Gamma(1 / 4)]^{4 / 3}} \frac{\hbar}{m a_{1}^{2 / 3}},
$$

beyond which the least bound $p$ wave molecular state is pushed to positive energy.

All higher branches of states in Figs. 3,5 are trap states. All lower branches, which approach those of molecular states in the absence of trapping [21], as discussed in the next section, are molecular states.

\section{Trapping shift of molecular spectrum}

Except for the least-bound state with binding energy comparable to or smaller than $\hbar \omega$, the effects of trapping on molecular states are generally weak, and can be treated perturbatively. The finite range of such states is such that the atoms in them would hardly feel the existence of a trap in their relative motion.

The nature of this perturbation is best understood by rewriting Eq. (33) for $e<0$ as

$$
\chi_{l}^{c(6)}\left(\epsilon_{s}\right)=K^{c}+\alpha_{l}^{(h o)}(e) \frac{W_{f+}^{c(6)}-K^{c} W_{g+}^{c(6)}}{W_{g-}^{c(6)}} .
$$

Comparing this equation to $\chi_{l}^{c(6)}\left(\epsilon_{s}\right)=K^{c}$, which determines the molecular spectrum in free space [21], the effect of trapping is isolated in this formulation to the second term in Eq. (52). From Eq. (37) and

$$
\xi_{l}^{(h o)}(e) \stackrel{-e \gg 1}{\longrightarrow} 1+\frac{1}{6} \frac{(l-1 / 2)(l+1 / 2)(l+3 / 2)}{|e|^{2}},
$$

it is clear, as expected, that the molecular states with binding much greater than $\hbar \omega$ are only weakly affected by the trapping, and can be treated by solving Eq. (52) perturbatively. To the lowest order in $\beta_{6} / a_{h o}$, the energy shift due to trapping is given by

$$
\Delta \epsilon_{l s}=q_{l}\left(\epsilon_{l s}\right)\left(\frac{\beta_{6}}{a_{h o}}\right)^{4}
$$


where

$q_{l}\left(\epsilon_{l s}\right)=(-1)^{l} \frac{(l-1 / 2)(l+1 / 2)(l+3 / 2)}{\left.6\left|\epsilon_{l s}\right|^{2}\left[W_{f-}^{c(6)} W_{g-}^{c(6) \prime}-W_{g-}^{c(6)} W_{f-}^{c(6) \prime}\right]\right|_{\epsilon_{s}=\epsilon_{l s}}}$,

and $\epsilon_{l s}=\epsilon_{l} / s_{E}$ is the scaled bound state energy of a molecule in the absence of the trap. Equation (54) means that trapping shift is, to the lowest order, proportional to $\left(\beta_{6} / a_{h o}\right)^{4}$, multiplied by a universal function of the scaled binding energy that goes to zero in the limit of $\left|\epsilon_{l s}\right| \rightarrow \infty$.

\section{E. Highly excited trap states}

For an highly excited trap state with $e \gg 1$, the determination of energy spectrum can also be further simplified. From

$$
\left.\xi_{l}^{(h o)}(e) \stackrel{e \gg 1}{\longrightarrow} \tan [\pi(e-l+1 / 2) / 2)\right],
$$

Eq. (46) reduces, for $e \gg 1$, to

$$
\tan [\pi(e-l+1 / 2) / 2]=-\tan \left[\delta_{l}(\epsilon)\right],
$$

or $(e-l+1 / 2) \pi / 2+\delta_{l}(\epsilon)=j \pi$, where $j$ is an integer. This result for $l=0$ has also been derived by Bolda et al. using a generalized pseudopotential [7]. It could also have been derived by using a semiclassical approximation for the harmonic part of the potential. For all energies of experimental interest, the phase shift $\delta_{l}$ can be accurately described using AQDT parametrization of Ref. 21].

\section{F. Long-range correlation between atoms in a trap}

In studies of quantum few-body and quantum manybody systems, it is often assumed that the wave function can be written in a Jastrow form [35], which is given, e.g., for bosons by

$$
\Psi=\left[\prod_{i=1}^{N} \phi\left(\mathbf{r}_{i}\right)\right] \prod_{i<j=1}^{N} F\left(r_{i j}\right) .
$$

Here $N$ is the number of particles, $\phi$ represents an independent-particle orbital, and $F$ is the pair correlation function. It is commonly assumed that $F$ has the asymptotic behavior of $F(r) \longrightarrow 1$ at large $r$, meaning that the particles become uncorrelated at large separations [35].

Our theory here provides a opportunity to check the validity of these assumptions, at least for $N=2$. To be specific, we restrict ourselves here to the lowest $s$ wave trap state for which the Jastrow assumptions are usually applied. Combining the wave function for the relative motion, as given by Eqs. (8)-(10), and that for the centerof-mass motion, which is a pure harmonic oscillator in its ground state, it is easy to show that the total wave function for two identical atoms in a symmetric harmonic trap can indeed be written in the Jastrow form but with a correlation function that behaves as

$$
F(r) \longrightarrow C r^{e-3 / 2},
$$

in the limit of large interatomic separations. Since $e$ can deviate significantly from $3 / 2$ for strong coupling $\left(a_{0} / a_{h o} \sim 1\right.$ or greater $)$, as illustrated in Fig. 3, this result implies that there exists significant long-range correlation for strongly-interacting particles in a trap. In a recent publication [24], we have shown that such longrange correlation exists not only for two particles in a trap, but also for $N(N>2)$ strongly-interacting particles in a trap.

\section{CONCLUSIONS}

In conclusion, we have presented a QDT for a harmonic potential and a two-scale QDT for two identical atoms in a symmetric harmonic trap. It is a general theory that is applicable to different partial waves, and from deeply bound molecular states to highly excited trap states. The only approximation in the theory, $\beta_{6} / a_{h o} \ll 1$, can be relaxed if needed. The result will still be of the form of Eq. (33) except that the $\chi^{(h o, 6)}$ function for $\beta_{6} / a_{h o} \sim 1$ or greater will require a more general solution of the twoscale potential described by Eq. (19). If the scattering length parameter used in our formulation is achieved by tuning around a Feshbach resonance [36, 37], the same results would apply, provided it is a broad Feshbach resonance with a width much greater than the energy scale $s_{E}$ associated with the van der Waals potential [38, 39, 40].

From a more general perspective, the theory we have presented here demonstrates the concept of multiscale QDT, which has potential implications for a range of problems. As examples, we mention two other two-scale potentials:

$$
V(r)=-C_{6} / r^{6}-C_{8} / r^{8},
$$

and

$$
V(r)=-Z / r-C_{4} / r^{4} .
$$

The former is of interest for a more accurate QDT description of long-range molecules over a wider range of energies, the latter is of interest for a more systematic understanding of the core polarization effect on atomic spectra. While such potentials have been well studied in numerical calculations, a more systematic approach to such two-scale problems may well be worthy of future efforts.

\section{Acknowledgments}

We thank Eite Tiesinga, Eric Bolda, and Paul Julienne for helpful discussions. This work was supported by the 
[1] U. Fano and A. Rau, Atomic Collisions and Spectra (Academic Press, Orlando, 1986).

[2] T. Busch, B.-G. Englert, K. Rzazewski, and M. Wilkens, Found. Phys. 28, 549 (1998).

[3] M. Olshanii, Phys. Rev. Lett. 81, 938 (1998).

[4] E. Tiesinga, C. J. Williams, F. H. Mies, and P. S. Julienne, Phys. Rev. A 61, 063416 (2000).

[5] D. Blume and C. H. Greene, Phys. Rev. A 65, 043613 (2002).

[6] M. Block and M. Holthaus, Phys. Rev. A 65, 052102 (2002).

[7] E. L. Bolda, E. Tiesinga, and P. S. Julienne, Phys. Rev. A 66, 013403 (2002).

[8] T. Bergeman, M. G. Moore, and M. Olshanii, Phys. Rev. Lett. 91, 163201 (2003).

[9] E. L. Bolda, E. Tiesinga, and P. S. Julienne, Phys. Rev. A 68, 032702 (2003).

[10] B. E. Granger and D. Blume, Phys. Rev. Lett. 92, 133202 (2004).

[11] H. Moritz, T. Stöferle, K. Günter, M. Köhl, and T. Esslinger, Phys. Rev. Lett. 94, 210401 (2005).

[12] T. Stöferle, H. Moritz, K. Günter, M. Köhl, and T. Esslinger, Phys. Rev. Lett. 96, 030401 (2006).

[13] Z. Idziaszek and T. Calarco, Phys. Rev. A 74, 022712 (2006).

[14] K. Huang and C. N. Yang, Phys. Rev. 105, 767 (1957).

[15] J. M. Blatt and D. J. Jackson, Phys. Rev. 76, 18 (1949).

[16] C. H. Greene, U. Fano, and G. Strinati, Phys. Rev. A 19, 1485 (1979).

[17] C. H. Greene, A. R. P. Rau, and U. Fano, Phys. Rev. A 26, 2441 (1982).

[18] B. Gao, Phys. Rev. A 58, 1728 (1998).
[19] B. Gao, Phys. Rev. A 59, 2778 (1999).

[20] A. L. Fetter and J. D. Walecka, Quantum Theory of Many-particle Systems (McGraw-Hill, New York, 1971).

[21] B. Gao, Phys. Rev. A 64, 010701(R) (2001).

[22] B. Gao, J. Phys. B 37, L227 (2004).

[23] B. Gao, Phys. Rev. Lett. 95, 240403 (2005).

[24] I. Khan and B. Gao, Phys. Rev. A 73, 063619 (2006).

[25] M. Abramowitz and I. A. Stegun, eds., Handbook of Mathematical Functions (National Bureau of Standards, Washington, D.C., 1964).

[26] B. Gao, unpublished.

[27] B. Gao, Phys. Rev. A 62, 050702(R) (2000).

[28] B. Gao, E. Tiesinga, C. J. Williams, and P. S. Julienne, Phys. Rev. A 72, 042719 (2005).

[29] B. Gao, Euro. Phys. J. D 31, 283 (2004).

[30] B. Gao, Phys. Rev. A 58, 4222 (1998).

[31] B. Gao, J. Phys. B 37, 4273 (2004).

[32] B. Gao, J. Phys. B 36, 2111 (2003).

[33] G. F. Gribakin and V. V. Flambaum, Phys. Rev. A 48, 546 (1993).

[34] B. R. Levy and J. B. Keller, J. Math. Phys. 4, 54 (1963).

[35] R. Jastrow, Phys. Rev. 98, 1479 (1955).

[36] W. C. Stwalley, Phys. Rev. Lett. 37, 1628 (1976).

[37] E. Tiesinga, B. J. Verhaar, and H. T. C. Stoof, Phys. Rev. A 47, 4114 (1993).

[38] T. Köhler, K. Góral, and T. Gasenzer, Phys. Rev. A 70, 023613 (2004).

[39] S. Simonucci, P. Pieri, and G. C. Strinati, Europhys. Lett. 69, 713 (2005).

[40] P. S. Julienne and B. Gao, physics/0609013. 